\title{
Linx
}

Revue des linguistes de l'université Paris X Nanterre

43 | 2000

Linguistique de l'écrit, linguistique du texte

\section{Cas de belligérance entre perspectives du narrateur et du personnage : neutralisation ou mise en résonance des points de vue?}

Conflicts between narratorial or actorial points of view: neutralization or hierarchization of the perspectives?

\section{Alain Rabatel}

\section{OpenEdition}

Journals

Édition électronique

URL : http://journals.openedition.org/linx/1095

DOI : 10.4000/linx.1095

ISSN : 2118-9692

\section{Éditeur}

Presses universitaires de Paris Nanterre

\section{Édition imprimée}

Date de publication : 1 décembre 2000

Pagination : 103-120

ISSN : 0246-8743

Référence électronique

Alain Rabatel, « Cas de belligérance entre perspectives du narrateur et du personnage : neutralisation ou mise en résonance des points de vue? », Linx [En ligne], 43 | 2000, mis en ligne le 11 juillet 2012, consulté le 19 avril 2019. URL : http://journals.openedition.org/linx/1095 ; DOI : 10.4000/linx.1095

Ce document a été généré automatiquement le 19 avril 2019

Département de Sciences du langage, Université Paris Ouest 


\section{Cas de belligérance entre perspectives du narrateur et $\mathrm{du}$ personnage : neutralisation ou mise en résonance des points de vue?}

Conflicts between narratorial or actorial points of view: neutralization or hierarchization of the perspectives?

Alain Rabatel

1 Nos travaux sur le point de vue (PDV) mettent en avant le concept de PDV représenté, en forte congruence avec celui de pensée représentée pour le DIL ${ }^{2}$. Dans Rabatel 1998, nous avons à plusieurs reprises souligné que la structure virtuelle du PDV permet d'interpréter, sur la base de mécanismes inférentiels, des situations où les paramètres habituels sont estompés (qu'il s'agisse de l'implicitation du procès perceptif ou du sujet à l'origine de la perception).

Restent les cas (c'est l'objet de ce travail) où des indices fonctionnent de manière contradictoire, provoquant des effets de brouillage du PDV. On se trouve alors face à des problèmes d'attribution, de délimitation, de détermination et de caractérisation des PDV. Comme les questions de délimitation des portées et d'enchâssements de PDV polyphoniques ont été abordées dans Rabatel 1998: 95-100 et 172-192, nous n'y reviendrons pas. Quant aux problèmes d'attribution concernant le focalisateur, ils feront l'objet d'une publication ultérieure, à partir du cas typique du statut de «on »; il en est de même pour les difficultés de caractérisation touchant aux effets de brouillage entre PDV représenté, raconté et asserté3 . Nous nous intéresserons donc ici aux difficultés de détermination naissant des conflits de perspective narrative, autrement dit de cas de belligérance entre PDV du narrateur et PDV du personnage. 


\section{0 . Bref rappel des paramètres du point de vue représenté}

3 Avant de traiter un exemple problématique, rappelons, à partir de deux courts exemples, les paramètres linguistiques sur lesquels se fonde le PDV représenté. Les énoncés en italiques de (1) et (2) correspondent non pas à une description « objective » du locuteurnarrateur, mais à une perception d'un énonciateur-personnage (autrement dit un focalisateur, ou un sujet de conscience) :

(1) GLEN promena son regard sur la place et les bâtiments en brique qui l'entouraient. Au centre, blanchi à la chaux, le vieux palais de justice où on l'avait condamné. Les voitures couvertes de poussières étaient garées en épi contre un trottoir très haut où circulaient des gens. «J'ai pas pris de p'tit déj. »"

(L. Brown, Père et fils, Gallimard La Noire 1999 : 10)

(2) La route de gravier serpentait vers une colline herbue, verte, luisante, rendue brûlante par le soleil de l'après-midi. Ils s'arrêtèrent à l'ombre des chênes et mangèrent, les portières ouvertes et la radio en marche.

«Tu vas te remettre avec elle, j'suppose ».

(ibid.: 15)

4 En (1), le focalisateur, mentionné par son nom propre, est à l'origine d'un procès de perception dénotant une perception soutenue dans le premier plan. Les éléments du second plan relèvent d'une progression thématique à thème éclaté, chaque objet $\mathrm{du}$ discours étant une partie du tout d'abord prédiqué dans son ensemble, dans le premier plan. L'usage de l'IMP donne à cette perception une coloration subjective: il faut comprendre que, certes, les voitures étaient garées en épi, que le palais de justice était blanchi à la chaux avant que Glen ne le " mentionne », mais que ces caractéristiques sont transmises au lecteur au moment où elles passent par le filtre perceptif de Glen ${ }^{5}$.

En (2), en dépit de l'absence explicite de NP et de procès de perception, la perception représentée est attribuée aux personnages contextuellement saillants ${ }^{6}$, Glen et son frère, Puppy. Cette inférence est justifiée par le fait que la situation (portières ouvertes) tout comme le site (face à une colline, en un lieu ombragé, propice à la perception) y conduisent, en vertu des inférences opérées par le lecteur à partir de son encyclopédie (intégrant la connaissance d'un certain nombre de topoï littéraires). Le fait que, dans nos deux exemples, les PDV soient suivis de paroles de personnages est bien évidemment un argument de plus en faveur de l'interprétation de ces énoncés descriptifs comme des PDV, mais c'est là un argument supplémentaire, et facultatif, pour ainsi dire, car l'interprétation serait la même si les fragments au discours direct étaient absents. Fondamentalement, en effet, ce qui justifie l'interprétation en termes de PDV, c'est le décrochage énonciatif.

6 Ces paramètres fonctionnent de manière satisfaisante lorsque les perceptions représentées renvoient à une origine énonciative ancrée dans un personnage, soit (à défaut de personnage focalisateur saillant) dans le narrateur. Mais, dès qu'on ne se 
contente pas d'extraits judicieusement découpés, les textes offrent des cas complexes, qui sont une occasion d'éprouver la validité du cadre théorique proposé :

(3) Mais ils s'arrêtaient quelquefois devant l'exposition complète de leur idée, et cherchaient alors à imaginer une phrase qui pût la traduire cependant. Elle ne confessa point sa passion pour un autre; il ne dit pas qu'il l'avait oubliée.

Peut-être ne se rappelait-il plus ses soupers après le bal, avec des débardeuses; et elle ne se souvenait pas sans doute des rendez-vous d'autrefois, quand elle courait le matin dans les herbes, vers le château de son amant.

(Flaubert, Madame Bovary, Le Livre de poche, 1983, p. 268s)

7 A plusieurs reprises, en (3), le narrateur épouse alternativement les perspectives des deux ex-amants, dans le premier comme dans le second plan. Dira-t-on qu'on se trouve face à deux PDV entrecroisés des personnages? Ce serait faire peu de cas de la présence du narrateur (sensible à travers les modalisateurs "peut-être » et «sans doute ", comme à travers l'effet qui résulte de ces demi-mensonges croisés). Préférera-t-on privilégier le point de vue du narrateur, qui refuse d'être la dupe des passions? Ce serait interpréter ces états instables comme des stratégies conscientes de tromperie de l'autre, alors que chacun se trompe lui-même aussi sûrement qu'il trompe son partenaire. (3) soulève donc des difficultés qui relèvent de l'analyse de la perspective, et, indissociablement, de l'interprétation qui en découle (ou qui la sous-tend).

Nous allons tenter de rendre compte des difficultés de détermination naissant de tels conflits. Ces cas de belligérance entre PDV du narrateur et du personnage ne portent pas seulement sur l'expression explicite de divergences axiologiques ou esthétiques, mais tiennent à des mécanismes scripturaux variés: combinaison syntagmatique de PDV divers (I), conflits entre sources évidentielles (II), ou brouillages affectant le débrayage énonciatif (III). Dans tous les cas que nous traiterons, le conflit opère sans annihiler l'une ou l'autre perspective : c'est pourquoi nous parlons d'effet de brouillage plutôt que de neutralisation des perspectives, ce qui présente l'avantage d'interpréter les textes en leur donnant une plus grande résonance, à rebours des inévitables réductions opérées par les entreprises typologisantes.

\section{L'interprétation de suites rapprochées (enchâssées ou enchaînées) de points de vue différents de personnages différents}

9 Il est rare qu'une œuvre adopte un point de vue unique, comme Ce que savait Maisy; au demeurant, cette perspective "unique » s'accompagne de relais de focalisation partiels sur d'autres personnages. Le plus souvent, les œuvres mêlent des PDV variables hiérarchisés. Cette situation peut s'entendre au moins de deux manières, l'une concernant des successions de PDV différents et (relativement) homogènes, l'autre concernant des suites très resserrées de PDV distincts et (relativement) hétérogènes. Faut-il interpréter ces suites rapprochées de PDV comme la marque d'une perspective du narrateur (Genette 1983: 49) ? Il n'y a pas de réponse tranchée à cette question, ainsi que le montre l'analyse de (4) et (5). 
10 (4) juxtapose spectaculairement les PDV des protagonistes de l'attentat des nationalistes contre l'archiduc d'Autriche, prélude à la première guerre mondiale : PDV de l'Archiduc (en italiques entre crochets), de la duchesse (en italiques), ainsi que de l'assassin, Princip (en caractères gras). Si l'on suivait Genette et ses épigones, (4) serait un récit à focalisation variable symptomatique de l'omniscience du narrateur. Pourquoi cette succession de PDV de personnages différents devrait-elle se convertir en une focalisation zéro (FZ), sauf à s'appuyer sur l'hypothèse, en l'occurrence non pertinente, de l'omniscience du narrateur? En effet, les perceptions et pensées plus ou moins construites ou fugitives des personnages sont transmises au lecteur par l'intermédiaire des personnages focaux, alors que le narrateur n'est sensible que dans la mise en scène du texte. Pour le reste, à aucun moment le narrateur ne manifeste qu'il en sait plus sur les personnages que les personnages eux-mêmes (à l'opposé des exemples de Hugo ou de Tolstoï). D'où ces successions de monologues intérieurs, composés parfois de phrases nominales exprimant l'entremêlement de pensées à peine ébauchées, dans le feu de l'action:

11 (4) François-Ferdinand s'est redressé à demi. [Qu'est-ce qu'il y a ? Potiorek [=le Gouverneur ] le gêne avec son dos. Au-dessus des têtes, il lit le nom du magasin qui fait l'angle. Machinalement. Siler. Les gens s'agglomèrent du côté droit. Ils ont reconnu la duchesse. Si on se décide pas à repartir, ça va être la cohue. On prendra du retard. Il a horreur de la cohue et d'être en retard. Quels que soient le lieu et l'horaire. A plus forte raison dans ce cul-de-sac. Le pire c'est à Vienne. Chaque année. Au moment de la procession de la Fête-Dieu sur le Graben. Marcher tête nue derrière l'Empereur. Lequel marchait derrière le dais. Tous ces gens aux balcons, retenus derrière des palissades décorées de guirlandes de verdure. Une double haie de hussards, avançant au pas lent du dais, sabre au clair. Soudain, ici, sur ce bord du quai, à l'angle de ce trottoir, il se sentit nu. Trop de monde. Pas de protection. Aucun ordre. La hiérarchie, l'étiquette, envolés. Tout à vau-l'eau. Même pas fichus de suivre un itinéraire! Un gouverneur incompétent. Des chauffeurs sourds. Un comte debout sur le marchepied. Le Wurstelprater. Mieux! Le Böhmischer Prater! A ce rythme, on servirait les soufflés froids et le Mostar chaud à l'hôtel Bosna.] La duchesse s'en fiche. Cet arrêt, ça l'amuse presque. Un blanc dans ses pensées tristes de la nuit. Elle voit des visages de près. D'enfants. De femmes. Elle sent des odeurs inconnues. Exotiques. Enivrantes? Elle descendrait bien marcher un peu. S'il n'y avait pas cette robe qui colle aux fesses à cause de la moleskine du siège. Elle soulève légèrement le - Derrière Potiorek, il l'a vu. Il les tient l'un et l'autre ! Qu'est-ce que c'est que ce couillon de Svaba sur le marchepied? Tant pis pour lui ? C'est... odmah! maintenant! Princip sort la main droite de sa poche. Lève lentement le bras. Très haut. (Sa veste ne le gêne pas, il a eu raison de -) L'abaisse. Tendu. Sutra. (demain) est là. Dans l'axe du bras. Demain est zelen, vert ! Il pense seulement ceci : levo (à gauche) Vrlo malo (très peu), car il sait que le pistolet porte à droite, et il presse l'index sur la détente. Une fois. 
BAUCH ! Il voit le cylindre jaune brillant de la douille sauter devant ses yeux. Raté ! Dans la portière. Cependant la duchesse a fait un bond en arrière en levant le bras droit. Merde! Il a touché la truie... Desno ! Desno ! (A droite! A doite!) Il entend GLING! la douille tomber sur le trottoir. L'Archiduc l'a vu. Il se dresse tout droit. Le fusille du regard. Son regard clair de chasseur, aux mille et mille pièces de gibier. Un regard de tueur. De connaisseur. Il sait qu'il va mourir. Qu'il meurt s'il - Il repousse sa femme des deux mains. Maintenant! Jos! (Encore! La duchesse s'affaisse contre l'Archiduc [...]
La duchesse sent le choc avant la brûlure, et la brûlure avant d'entendre le sifflement FELLENT! de la balle, en même temps que l'impact DUNG! de celle-ci dans le métal de la portière. Une douleur fulgurante dans le ventre. Du côté droit. Elle ouvre les yeux Se sent irrésistiblement poussée contre les cuisses de Frantisek I= appellatif affectueux de son mari, l'Archiduc «François »- Joseph]. Frantisek s'est levé. Elle voit. (il ne faut pas !) la gueule noire si petite, si ronde, gentille en soi, innocente, de l'arme pointée. Elle sent que Frantisek essaie de se dégager. Il la repousse sans ménagement. Elle voudrait l'aider. S'alléger. Elle ne peut pas. [...]

(J. Guerreschi, Montée en première ligne, Presses Pocket, p. 136 s.)

Le critère décisif, c'est le mode de donation des référents, et, conjointement, la détermination de l'instance énonciative susceptible de l'assumer. En (4), toutes les informations passant uniquement par le filtre des personnages focalisateurs, le récit imbrique leurs PDV, sans créer de FZ. Par conséquent, il faut relativiser l'analyse de Todorov selon laquelle

Un seul personnage peut être vu «du dedans» (et cela mène à la «focalisation interne ») ou tous - ce qui produit le récit « à narrateur omniscient »

(Todorov 1973: 61)

13 Cette formulation pêche par excès : l'omniscience du narrateur n'est pertinente que pour autant que texte la manifeste linguistiquement, ce qui est loin d'être toujours le cas.

Il existe néanmoins des contre-exemples proches de l'exemple précédent, où, pourtant, il se produit de facto une impression d'omniscience narratoriale. Des successions de PDV de divers personnages deviennent par simple contiguïté des marques de l'omniscience du narrateur, lequel se borne à accéder aux pensées de plusieurs personnages au même moment, en des lieux différents, sans manifester autrement sa supériorité cognitive au détriment des personnages. C'est apparemment la seule différence avec (4) :

(5) Un clodo frissonnant, soulagé de trouver un lit à sa convenance, s'allongea sur un banc de la place Sainte-Marthe, tout près de l'endroit où l'on avait découvert le cadavre le matin même. Il était deux heures. Un nuage masqua le quartier de lune, et bientôt il plut. Recroquevillé sur son lit de fortune, le clodo leva un poing vengeur vers le ciel dans un geste emphatique.

Maryse Horvel dodelinait de la tête, heureuse et alanguie, en caressant la tête de Butch, enfouie entre ses cuisses. Elle oubliait la putain du périphérique, la petite vieille poignardée square Réaumur, toute cette galerie des horreurs ordinaires, consignée sur les registres de la huitième section du parquet, et s'adonnait à son plaisir.

Rue de Tourtille, Nadia savourait sa première soirée de tranquillité. [...] 
Il était deux heures du matin. Une pluie fine tombait sur toute la région parisienne. Rovère se servit un dernier verre et le but d'une traite. Après quoi, en bras de chemise, dédaigneux du crachin, il sortit dans le jardin de son pavillon, tout près de la Croix-de -Chavaux. [...]

(Th. Jonquet, Les orpailleurs, Série Noire Gallimard, 1993, p. 64 et 65) personnages, au même moment et en des lieux distincts qu'au fait qu'il matérialise ces informations dans l'espace resserré du texte. On assiste à la construction d'un éthos narratorial omniscient, par la mise en scène du savoir dans l'espace de l'énonciation fictive, à travers sa matérialisation sous la forme d'une forte densité et contiguïté spatiale. Cette hypothèse est linguistiquement confirmée par la valeur des PS, qui ne sont pas ici des formes de visée globale: ces actions sont simultanées, et construisent de ce fait des seconds plans chronologiques, entrant dans une construction thématique à thème éclaté consacrée aux moments d'intimité des protagonistes de l'affaire. Une interprétation qui privilégierait exclusivement l'une ou l'autre hypothèse (FZ seule ou focalisations internes successives sans FZ) priverait le texte de l'effet de résonance opéré par cette mise en relation de perspectives hiérarchisées rendant compte de la diversité des forces qui meuvent des personnages au vécu si divers, par ailleurs réunis par le drame et par leur métier, comme par leur difficile quête du bonheur (c'est la perspective du narrateur qui nous le fait entendre).

Si la notion de focalisation variable n'est pas contestable, elle n'est pas pertinente pour donner infailliblement un contenu à la $\mathrm{FZ}^{7}$, comme on va le vérifier à partir d'un autre cas de brouillage résultant de tensions contradictoires entre perspectives du personnage et du narrateur.

\section{Effets de belligérance entre percevoir/savoir du personnage et percevoir/savoir du narrateur}

17 Ces effets se rencontrent lorsque les textes opèrent un débrayage énonciatif, et que la référenciation $\mathrm{du}$ focalisé comporte des informations, des manières de voir qui, manifestement, ne sont pas celles de l'instance focalisatrice :

(6) La seconde année, précisément au point de cette histoire où le lecteur est parvenu, il arriva que cette habitude du Luxembourg s'interrompit sans que Marius sût trop pourquoi lui-même, et qu'il fut près de six mois sans mettre les pieds dans son allée. Un jour enfin il y retourna. C'était par une sereine matinée d'été, Marius était joyeux comme on l'est quand il fait beau. Il lui semblait qu'il avait dans le coeur tous les chants d'oiseaux qu'il entendait et tous les morceaux du ciel bleu qu'il voyait à travers les feuilles des arbres.

Il alla droit à "son allée ", et, quand il fut au bout, il aperçut, toujours sur le même banc, ce couple connu. Seulement quand il approcha, c'était bien le même homme ; mais il lui parut que ce n'était plus la même fille. La personne qu'il voyait maintenant était une grande et belle créature ayant toutes les formes les plus charmantes de la femme à ce moment précis où elles se combinent encore avec toutes les grâces les plus naïves de l'enfant; moment fugitif que seuls peuvent traduire ces deux mots : quinze ans. 
(Hugo, Les Misérables Folio, Gallimard, T. II, p 286)

18 D'une part, l'apparition de "mademoiselle Lanoire " et de "monsieur Leblanc » est d'abord présentée à travers le PDV de Marius, ancré par le verbe de perception «il aperçut ", puis représenté par les imparfaits de second plan à valeur anaphorique méronomique (chacun des deux est une partie de ce tout qu'est le «couple»), ainsi que par la corrélation des imparfaits et de "maintenant », particulièrement efficace pour l'expression des pensées au DIL (« c'était bien le même homme »).

D'autre part, cet extrait illustre bien une supériorité du PDV du narrateur sur celui du personnage. Les deux PDV sont à ce point entremêlés qu'ils apparaissent dans les mêmes phrases : ainsi, alors que le lecteur voit la scène avec les yeux de Marius, notamment par le biais du discours intérieur, les verbes d'attitude propositionnelle " il lui semblait », « il lui parut » précisent que le narrateur est dans une situation de supériorité cognitive sur son personnage, dans la mesure où ce dernier voit des signes qu'il interprète mal, à la différence du narrateur.

De sorte que la situation peut se résumer ainsi : c'est Marius qui "voit» (et qui sait, dans une moindre mesure, c'est-à-dire qu'il croit savoir) alors que c'est le narrateur qui "sait " .Certes, ce voir et le savoir tout relatif qu'il indique sont embryonnaires, et sous la dépendance du savoir du narrateur, mais ils existent bel et bien. Il y a là une situation, somme toute banale, mais que Genette et ses successeurs (sauf $F$. Jost) n'aident guère à penser, puisque la coutume est de considérer que la source du "voir » et du «savoir » serait toujours ancrée homogènement et exclusivement dans la même instance.

(6) serait-il une FZ ou une focalisation interne? Les deux termes de l'alternative conduisent chacun à une regrettable mise à plat de ce fragment, dont le charme provient du jeu inégal entre le voir et le savoir du narrateur et du personnage. Il est plus opératoire de dire que le PDV de Marius (repérable par sa dimension essentiellement perceptive) est dominé par le PDV du narrateur (caractérisé essentiellement par sa dimension cognitive). D’ailleurs, les commentaires du narrateur, en début de chapitre, sur la conduite du récit, le fait qu'il précise que Marius ne sait pas expliquer le sens de son changement de comportement, l'ellipse temporelle, ou encore le commentaire final sur cette métamorphose de Cosette, que ce jeune homme est incapable d'analyser, tout cela construit cette incessante mise en scène du savoir du narrateur, exhibé par les titres du chapitre ou du livre («La conjonction de deux étoiles ») : ce phénomène "parasite» même la vision de Marius, comme l'indiquent les guillemets entourant la deuxième occurrence de "son allée ", comme s'ils dénotaient toute la distance qui sépare celui qui sait (le narrateur) du jeune puceau. (7) présente un cas de figure similaire :

(7) Depuis longtemps déjà elle le voyait et elle l'examinait comme les filles examinent et voient, en regardant ailleurs. Marius trouvait encore Cosette laide que déjà Cosette trouvait Marius beau. Mais comme il ne prenait point garde à elle, ce jeune homme lui était bien égal.

Cependant elle ne pouvait s'empêcher de se dire qu'il avait de beaux cheveux, de beaux yeux, de belles dents, un charmant son de voix quand elle l'entendait causer avec ses camarades, qu'il marchait en se tenant mal, si l'on veut, mais avec une grâce à lui, qu'il ne paraissait pas bête du tout, que toute sa personne était noble, douce, simple et fière, et qu'enfin il avait l'air pauvre, mais qu'il avait bon air.

Le jour où leurs yeux se rencontrèrent et se dirent enfin brusquement ces 
premières choses obscures et ineffables que le regard balbutie, Cosette ne comprit pas d'abord.

(Hugo, Les Misérables, Folio, Gallimard, T. II, p. 493s) dernier donne la signification exacte des événements, voit dans l'âme et le cœur des personnages mieux que les principaux intéressés: en témoigne notamment le jeu des adverbes "enfin » et "d'abord ", dans la dernière phrase, comme si le narrateur était impatient de voir Cosette percer les secrets de l'amour. Cet effet de supériorité cognitive du narrateur est renforcé par le commentaire amusé sur le comportement des filles qui examinent « comme les filles examinent et voient, en regardant ailleurs », et par l'ironie que l'on peut déceler au travers du «cependant » du deuxième paragraphe, qui rend manifeste la contradiction entre l'indifférence affichée de Cosette pour Marius, et les mille détails qui révèlent l'attention passionnée qu'elle a pour lui (elle est non seulement sous le charme du physique et de l'apparence générale du jeune homme, mais encore de sa voix « quand elle l'entendait [il faut comprendre : quand elle l'écoutait] causer avec ses camarades »).

On pourrait penser que ce brouillage des perspectives est inévitable, avec les verbes de procès mental, et que ce n'est pas le cas avec des verbes de perception. Cette distinction n'est pas sans valeur, mais, comme on va le voir, à la condition expresse qu'on ne la dichotomise pas. Tout verbe de perception, du moins sous sa forme affirmative, indique que le savoir porte essentiellement sur l'objet considéré ( $P$ Pierre vit $P$ ») et secondairement sur la manière dont le sujet perçoit ( Pierre détaillait $P$ »). Mais le narrateur n'est pas nécessairement présent, de sorte que les informations sur les perceptions du personnage-focalisateur viennent quasi immédiatement au lecteur, sauf cas de modalisation ("Pierre croyait voir $P$ », «Pierre devait entendre $P$ ", "Pierre pensait sentir $P »)$, ou tournure négative (« Pierre n'entendait pas $P »)$.

Il serait néamoins erroné de conclure que toute négation implique un PDV : cela ne se produit que si la négation porte sur l'acte de perception et non sur l'objet sur lequel porte l'acte de perception - effectué à défaut d'avoir atteint la cible attendue :

(8) Il [ =Glen] s'arrêta au milieu du cimetière et jeta un regard circulaire. Puppy avait dit pas loin de tante Eva, mais il n'était pas sûr de savoir où elle était. Ça faisait si longtemps qu'elle était morte. L'enterrement d'Eva était une vieille histoire, il s'en souvenait à peine. Des gamins avec des cravates, des femmes en pleurs, et de la boue sur les chaussures. Il était petit à l'époque. Un Davis, ou un Clark, elle serait près d'eux. Il se mit à lire les noms en progressant vers la droite, puis il se trouva brusquement parmi eux. Ils étaient tous enterrés ensemble, et cela depuis cent ans. Les pères, les mères, les enfants, les grands-pères et les victimes de trois guerres. Il trouva la tombe, et il fut stupéfait. Pas de pierre tombale, rien qu'un petit écusson en métal avec une carte blanche fixée dessus par une pince. Le nom de la société de pompes funèbres était marqué en relief, et c'était ce qui désignait l'endroit où elle reposait.

(L. Brown, op. cit. : 17)

(9)Résolu à ne rien voir, il [Alexis Alexandrovitch Karénine] ne s'apercevait pas que bien des personnes battaient déjà froid à sa femme; résolu à ne rien 
approfondir, il ne se demandait pas pourquoi celle-ci avait voulu s'installer à Tsarkoié où demeurait Betsy et non loin du camp de Vronski.

(Tolstoï, Anna Karénine, Folio, Gallimard, p. 228)

(10)Pierre ignorait seulement que le village où on lui avait offert le pain et le sel et où on bâtissait une chapelle en l'honneur des saints et des apôtres, était un bourg commerçant où se tenait une foire à la Saint-Pierre et que la chapelle avait été commencée depuis longtemps par les riches paysans du lieu, ceux précisément qui l'avaient accueilli, mais que les neuf dixièmes des habitants de la localité étaient complètement ruinés. Il ne savait pas que si, conformément à ses ordres, on avait bien exempté des corvées au-dehors les femmes ayant des enfants en bas âge, ces mêmes femmes étaient d'autant plus surchargées de travail à la maison. Il ne savait pas que le prêtre qui l'avait reçu la croix à la main accablait les paysans de redevances, que les parents lui amenaient leurs enfants en pleurant et ne les récupéraient que contre le versement d'une forte somme. Il ne savait pas que les édifices de pierre construits selon ses plans étaient bâtis par les paysans euxmêmes, ce qui aggravait la corvée, réduite uniquement sur le papier. Il ne savait pas que là où le régisseur lui montrait d'après les livres que les redevances avaient été diminuées d'un tiers, conformément à ses instructions, les corvées avaient été augmentées du double. Aussi Pierre fut-il ravi de son voyage et il retrouva les dispositions philanthropiques dans lesquelles il était en quittant Petersbourg.

(Tolstoï, La Guerre et la Paix, Folio, Gallimard T. I, p. 490) active d'abord de vagues souvenirs de l'enterrement de tante Eva. Il passe de perceptions représentationnelles, remémorées, à des perceptions actuelles, en cherchant la tombe de sa mère à partir d'indices (s'il y a les tombes de Clark ou de Davis, alors la tombe ne sera pas loin du carré où se trouvent tous les morts de la famille). Glen s'attend à trouver la pierre tombale de sa mère, et constate qu'il n'y a à la place qu'une pauvre croix de bois. L'acte de perception est réussi, mais l'objet que Glen s'attendait à voir manque : cette négation n'infirme donc pas le PDV de Glen. Il n'y a rien de commun avec les négations de (9) et de (10), puisque ce qui est nié, c'est l'acte même de perception ou de cognition, que le héros n'accomplit pas. Les fragments en caractères gras indiquent l'ignorance (volontaire ou involontaire, peu importe, en l'occurrence) des personnages. Dans ces conditions, le savoir, dont il est ici fait état, est transmis au lecteur par l'intermédiaire du narrateur, qui manifeste linguistiquement sa supériorité sur le personnage. Par voie de conséquence, le brouillage entre PDV du personnage et PDV du narrateur est moindre en (9) et (10) qu'en (6) et (7), alors qu'il n'y en a aucun en (8).

Si l'activité de perception peut être présentée de telle manière que l'information vienne au lecteur sans l'intermédiaire du narrateur, en revanche, l'information sur les pensées du personnage suppose toujours l'existence de ce même narrateur, quand bien même il se fait le plus discret possible. Derrière tout énoncé du type «Pierre (verbe de procès mental) $P$ », il y a toujours un narrateur implicite qui présuppose que ce que Pierre pense est juste (" Pierre savait que $P$ ) ou faux ( Pierre croyait que $P »)$; partiel ( Pierre entrevit que $P »)$ ou global (« Pierre comprit que $P »)$, subjectif («Pierre s'imaginait que $P »)$ ou objectif (« Pierre admettait que $P »)$, etc. En ce sens, la part informative concernant les pensées du personnage porte essentiellement sur le personnage, et secondairement sur le regard que 
le narrateur porte sur le personnage, garantissant ou non l'exactitude du savoir véhiculé, etc ${ }^{8}$. Bref, la vérité d'une perception du personnage n'est pas nécessairement garantie par le narrateur, tandis que celle d'une pensée du personnage renvoie nécessairement à la parole autorisée du narrateur. Autrement dit, le savoir que le lecteur apprend des perceptions du personnage est relativement autonome par rapport au savoir du narrateur ', et c'est ce qui explique que les perceptions soient si souvent prises à la lettre pour la vérité, dans le cadre du réalisme. En revanche (nous ne disons pas : à l'opposé), le savoir que le lecteur acquiert sur les pensées du personnage est toujours de surcroît un savoir du narrateur sur le personnage. Il y a donc un déséquilibre entre les informations véhiculées par les verbes de perception et de procès mental.

Il en résulte que le PDV du personnage est plus nettement homogène et repérable quand il porte sur des perceptions, et qu'il est au contraire plus difficilement isolable quand il porte sur des pensées, puisque les pensées n'existent le plus souvent que rapportées par le narrateur, confirmées ou infirmées, appréciées, moquées, mises à distance, etc. Cette conclusion est cependant à moduler fortement : en effet, comme le montre la proximité du PDV et du DIL, il est illusoire de prétendre que la perception puisse être découplée d'une quelconque activité de cognition (ou de pensée) ${ }^{10}$ : en sorte que notre conclusion précédente est à prendre pour ce qu'elle est, une approximation commode, qu'il serait oiseux de prétendre ériger en dogme.

\section{Brouillage sur l'opposition fonctionnelle des plans et sur les personnes}

L'effet de brouillage entre perspectives narratives différentes est maximal avec l'estompage du débrayage énonciatif. Dans les récits hétérodiégétiques ce phénomène concerne l'opposition fonctionnelle des plans (Rabatel 1998: 36); dans les récits homodiégétiques (fictifs ou non), ce brouillage aspectuel est redoublé d'un brouillage sur les personnes, le syncrétisme du sujet parlant, du locuteur et de l'énonciateur y masquant les différences de rôles.

(11)Il fait chaud, encore plus chaud que l'an dernier, Hermantier, dans l'allée du jardin, enlève ses lunettes, offre au soleil sa face mutilée. C'est encore une joie de sentir le long de sa peau courir ce petit vent sec, qui sent le miel et la rose. Il y a des insectes qui passent en ronflant et, parfois une guêpe - c'est sans doute une guêpe - tourne autour de son visage, cherchant à se poser. Il descend l'allée, tranquillement, les mains dans les poches, s'efforçant d'être naturel, de ne pas se vo ûter, de ne pas, non plus, renverser la tête en arrière. Le plus dur c'est de marcher sans penser qu'on marche et d'avancer sans s'imaginer qu'on va heurter un mur. $\mathrm{Au}$ début, il était hanté par la peur du mur; il avait toujours envie de tendre les bras en avant, et quelque chose se recroquevillait dans sa poitrine. Tout son corps était comme une bête peureuse. On a beau décider qu'il n'y a pas d'obstacle, le ventre, les genoux n'obéissent plus et, déjà, se garent, se préparent à la douleur du choc. On a sans cesse l'impression que l'air est plus dense. Comme au voisinage d'une paroi. Hermantier était obligé de s'arrêter souvent pour faire le point. "Je suis à une vingtaine de pas de la terrasse, bon. Je suis au large. La grille est encore loin ». Peu à peu il reprenait confiance. Il se guidait à l'oreille. Quand ses semelles ne faisaient plus craquer le gravier, c'est qu'il obliquait vers le parterre. Il n'arrivait pas à suivre une direction rectiligne. Toujours, il venait à gauche, comme un voilier vicieux. La traversée du jardin était une épuisante équipée.

Maintenant, ses pieds commencent à connaître les détours des allées, à condition de 
parcourir toujours le même itinéraire. Curieux comme on prend possession des choses, quand on ne les voit plus.

(Boileau-Narcejac, Les visages de l'ombre, Bouquins, T. I, Laffont, p. 231s) narrateur se garde bien de garantir les informations. Le suspens du pacte fiduciaire provient du brouillage des plans, qui apparait on ne peut plus nettement dans la phrase où Hermantier descend l'allée, et s'efforce d'être naturel. Cette phrase peut aussi bien être un commentaire du narrateur qu'un fragment de soliloque, et, dans ce cas, les gestes " décrits » sont à interpréter comme des ordres que le focalisateur s'adresserait à luimême : "sois naturel, ne te voûte pas, renverse ta/la tête en arrière ". Cette hésitation contribue au suspens savamment entretenu sur l'état physique du personnage... puisque le récit a la rouerie de s'écrire sous la vision et la visée du personnage.

En raison de ses nombreuses valeurs, le présent se prête particulièrement bien à l'expression des perceptions et/ou des pensées représentées en relation plus ou moins lâche avec les événements du premier plan, et joue un rôle majeur dans les monologues intérieurs, comme en (12), qui exprime les réactions d'une vieille grand-mère au terme de sa vie, face à la lutte désespérée de son petit fils contre le choléra :

(12) On fait sa vie. Il faut vouloir sa vie. La volonté d'aimer, de vivre, est un arbre naturel, vigoureux, qui vous pousse dans le corps. L'existence est ce qu'elle est. Les hommes sont ce qu'ils sont. Le mieux est toujours quelque part. Dans le sable, dans le granit, dans le plomb, en nous-mêmes. Le don des larmes, la grâce des larmes est toujours quelque part.

Comme elle éprouve son vieux corps, comme elle sent sa vieille âme, toute pétrie de passé. Comme tout remue en elle. Mille vies se contredisent à l'intérieur de sa seule vie. L'âme qui renonce et celle qui poursuit sont les siennes ; l'âme quotidienne et celle qui regarde au loin. L'âme des colères est la sienne et puis aussi l'âme de la plus secrète douceur.

Tout s'apaise après qu'on a longtemps pleuré. En pressant ses deux paumes contre ses yeux, puis les écartant comme deux ailes vers les tempes, Om Hassan essuie son visage. Avant de se pencher de nouveau sur l'enfant, elle efface toute trace de pleurs. Et même, elle dissimule sous son voile une mèche blanche ; Hassan en serait sans doute bouleversé, il n'a jamais vu sa grand-mère en cheveux. Toujours assise, elle s'approche de la cachette.

Elle a soulevé une fois encore le tissu qui recouvre le réduit. Rien n'a changé, pourtant tout est différent.

Ces marbrures, cette sueur sont des vêtements d'emprunt. Ce souffle bruyant n'est pas le signe de la fin, mais celui du grand combat; et rien ne se gagne sans combat. Cette chair, ces os rassemblés ne sont pas vraiment Hassan. Hassan est derrière tout cela, qui veille. L'enfant lui-même ne semble pas tellement y croire, à son propre corps. Malgré ce corps, il va vivre. Les enfants des hommes font de ces miracles; pas les poupées. Hier, n'a-t-il pas demandé : «On part...? ? Il sait qu'on se dirige vers la mer. Il veut voir la mer. Il la verra.

Un grand vent balaye les incertitudes et les tristes souvenirs. Il n'y a plus sa mère confondue aux murs, mais sa mère qui rit au crépuscule tandis que les hommes rentrent des champs; et puis il y a son père qui vient d'acheter son premier feddan. Il y a la lune du soir où Saïd l'a aimée. Il n'y a pas seulement la cueillette du coton, à six ans, courbée sous le soleil en délire [...].

(A. Chédid, Le sixième jour, Librio, 1986, p. 108) 
31 Les présents de (12) expriment soit le premier plan (« essuie », « s'approche », etc.) soit, le plus souvent, des perceptions étroitement associées aux pensées («ce souffle bruyant n'est pas le souffle de la fin ", etc.), qui peuvent s'épanouir en un véritable monologue lyrique. Cette forme verbale est si plastique que les commentaires d'ordre général qu'Om Hassan tire de son expérience (paragraphes 1 et 2) pourraient tout aussi bien être attribués au narrateur, dans le cadre d'un récit consonant ${ }^{11}$. Et ce n'est pas le moindre intérêt de cette forme verbale que de se prêter si facilement à l'expression des pensées présentes ou passées, ou atemporelles, ainsi qu'à l'expression de pensées personnelles ou générales, par d'imperceptibles transitions...

Par conséquent, l'utilisation du présent (même si elle ne neutralise pas totalement l'opposition fonctionnelle des plans, en vertu du fait que l'utilisation d'un même tiroir temporel ne neutralise pas les différences de visée) estompe la frontière entre perceptions représentées et pensées représentées, autrement dit entre ce qui, chez Genette, relève du mode narratif (le PDV) et de la voix (le monologue intérieur), et donc est susceptible d'affecter le choix d'une perspective narrative.

Ce phénomène, plus net avec les récits homodiégétiques, pose de redoutables questions à l'interprétation. En effet, s'il est théoriquement indispensable de dissocier je-narré (jepersonnage) et je-narrant, pratiquement, cette distinction n'est pas toujours nette :

(13) La cloche de minuit m'a éveillé. Affreux réveil lorsqu'il fait regretter le rien ou les illusions du sommeil. Je ne pouvais pas croire d'avoir passé trois heures sans avoir senti aucun mal. Sans bouger, couché comme j'étais sur mon côté gauche, j'ai allongé le bras droit pour prendre mon mouchoir que la réminiscence me rendait sûr d'avoir placé là. En allant à tâtons avec ma main, Dieu ! quelle surprise lorsque j'en trouve une autre froide comme glace. L'effroi m'a électrisé depuis la tête jusqu'aux pieds, et mes cheveux se hérissèrent : jamais je n'ai eu dans toute ma vie l'âme saisie d'une telle frayeur, et je ne m'en suis jamais cru susceptible ; j'ai passé certainement trois ou quatre minutes non seulement immobile, mais incapable de penser ; rendu à moi-même je me suis fait la grâce de croire que la main que j'avais touchée n'était qu'un objet de l'imagination. Dans cette ferme supposition j'allonge de nouveau le bras au même endroit, et je trouve la même main, que jetant un cri perçant et transi d'horreur je serre, et je relâche en retirant mon bras. Je frémis ; mais devenu maître de mon raisonnement je décide que pendant que je dormais on avait mis près de moi un cadavre. [...] Cette pensée me rend féroce : je porte pour la troisième fois mon bras à la main, je la saisis, je la serre, et je veux dans le même instant me lever pour tirer à moi ce cadavre, et me rendre certain de toute l'atrocité de ce fait : mais voulant m'appuyer sur mon coude gauche, la même main froide que je tenais serrée devient vive, se retire, et je me sens dans l'instant avec ma grande surprise convaincu que je ne tenais dans ma main droite aucune main que ma même main gauche, qui percluse et engourdie avait perdu mouvement, sentiment et chaleur, effet du lit tendre, flexible et douillet sur lequel mon pauvre individu reposait

(Casanova, Histoire de ma fuite des Plombs, 10/18, 1999: $30 \mathrm{~s}$ )

Cette narration ultérieure entremêle des considérations renvoyant autant au je-narré qu'au je-narrant, Casanova restituant avec vivacité (par le biais des présents qui se substituent aux formes de PC et de PS) la prégnance des sensations passées. Comme par ailleurs le récit est homodiégétique, la frontière entre le mode et la voix s'estompe, et l'on ne sait plus vraiment si l'émotion est celle du Casanova prisonnier d'autrefois, ou du Casanova écrivant. On pourrait objecter que l'on se trouve face à une hypotypose, bref, à 
un fait de style sans grand enjeu interprétatif. Soit. Mais que dira-t-on des efforts de Casanova pour maîtriser sa peur, racontés eux-aussi au présent? S'agit-il d'efforts du jenarré, ou, plutôt, du je-narrant? Ce courage et cette clairvoyance datent-ils de l'époque des faits, ou proviennent-ils du confort que procure la distance et l'absence du danger? Cette question pèse plus lourd que la précédente, sans doute parce qu'elle porte sur des phénomènes interprétatifs alors que la première ne mettait en jeu que la vivacité de phénomènes perceptifs. On est à la charnière de problèmes comportant de vrais enjeux axiologiques, idéologiques, tels qu'il s'en produit dans les textes de fiction ( $L a$ vie de Marianne repose entièrement sur cette ambiguité-là) ou dans les autobiographies : et, si la place ne faisait défaut, il ne serait pas difficile de montrer que maints mémorialistes acteurs importants des événements jouent (volontairement ou non) sur cet effet de brouillage-là ${ }^{12}$.

Si, dans les conventions du réalisme, les sources perceptives et cognitives du PDV vont souvent de pair, ce n'est pas toujours le cas, non seulement dans les cas de dissonance ironique, mais encore dans les cas de "belligérance » entre perspectives, dont on a vu qu'elles ne reposaient pas seulement sur des divergences axiologiques explicites, mais encore sur des mécanismes linguistiques parfois ténus comme le choix des verbes de perception ou de procès mental, la négation, le jeu sur l'aspect ou la personne, etc.

Il existe bien des PDV du personnage ou de narrateur homogènes ; mais il en existe tout autant qui ne le sont pas. Dans ces conditions, privilégier une perspective à l'exclusion de l'autre, c'est opérer une réduction navrante, et se priver des délices de l'interprétation, des mises en résonance qui participent d'une polyphonie étendue. Dans tous les cas, les effets de sens mettent à contribution les beautés grammairiennes de la langue, et jouent sur les liaisons et déliaisons constitutives de l'aventure littéraire, sans que le fonctionnement souple de la structure virtuelle du PDV ne soit mis à mal.

\section{BIBLIOGRAPHIE}

BALLY C. 1912 a « Le style indirect libre en français moderne, I », Germanische Romanische Monatschrift, fas. 4, 549 à 556.

BALLY C. 1912 b « Le style indirect libre en français moderne, II », Germanische Romanische Monatschrift, fas. 4, 597 à 608.

BALLY C. 1914 " Figures de pensée et formes linguistiques », Germanische Romanische Monatschrift, fas. 6, 405 à 422 et 456 à 470 .

BANFIELD A. 1995 Phrases sans parole. Théorie du récit et style indirect libre. Le Seuil, Paris.

BENZAKOUR F. 1990 Les compléments de comptes rendus de perception : quelques cas en français. Thèse de doctorat, Strasbourg II.

BERTHONNEAU A.-M. KLEIBER G. 1993 « Pour une nouvelle approche de l'imparfait : l'imparfait, un temps anaphorique méronomique ", Langages no 112, 55 à 73. 
COMBETTES B. 1992 L'Organisation du texte. Metz, Université de Metz, Centre d'analyse Syntaxique de l'Université de Metz.

DENDALE P. TASMOWSKI L. «L'évidentialité ou le marquage des sources du savoir », Langue française $\mathrm{n}^{\circ} 1021994$. Paris Larousse.

DUCROT O. 1984 Le Dire et le Dit. Paris, Minuit.

DUCROT O. 1989 Logique, structure, énonciation. Paris, Minuit.

GENETTE G. 1972 Figures III. Paris, Le Seuil.

GENETTE G. 1983 Nouveau discours sur le récit. Paris, Le Seuil.

GENETTE G. 1991 Fiction et diction. Paris, Le Seuil.

MARNETTE S. 1998 Narrateur et points de vue dans la littérature française médiévale. Une approche linguistique. Berne, Peter Lang.

RABATEL A. 1997a «L'introuvable focalisation externe », Littérature n 107. Larousse, Paris.

RABATEL A. 1997b Une histoire du point de vue. Klincksieck, Paris/Centre d'Etudes Linguistiques des Textes et des Discours. Université de Metz.

RABATEL A. 1998 La construction textuelle du point de vue. Paris, Lausanne, Delachaux et Niestlé.

RABATEL A. 1999 « Mais dans les énoncés narratifs : un embrayeur du point de vue et un organisateur textuel », Le français moderne, vol 1.

RABATEL A. « Fréquence itérative et perspective narrative : un retour critique sur l'omniscience narratoriale et sur la restriction de champ du personnage » (à paraitre).

RABATEL A. 2000a « Un, deux, trois points de vue ? Pour une approche unifiante des points de vue narratifs et discursifs ", La lecture littéraire, $\mathrm{n}^{\circ} 4$.

RABATEL A. 2000b « Quand voir c'est (faire) penser ». Actes du colloque « Les relations intersémiotiques ». Lyon, 16/18 décembre 1999, Panier L. (éd.).

ROSIER L. 1999 Le discours rapporté. Histoire, théories, pratiques. Bruxelles, De Boeck-Duculot.

VOGELEER S. «L'accès perceptuel à l'information : à propos des expressions un homme arrive/on voit arriver un homme ", Langue française $\mathrm{n}^{\circ} 102$, 1994, Larousse, Paris.

\section{NOTES}

1. Le PDV est en première approximation un parasynonyme de la notion de focalisation chez Genette.

2. Si le PDV est proche du DIL, en vertu du fait que les perceptions s'accompagnent toujours plus ou moins d'un procès mental (c'est pourquoi nous parlons de perception et/ou de pensée représentées). Il existe malgré tout des perceptions représentées très peu colorées par de la pensée indirecte libre : c'est le cas en (1) et (2). Proximité ne signifie pas égalité : on distinguera le DIL du PDV par le fait que ce dernier intrique nécessairement perceptions et pensées, alors que le DIL peut se limiter à des pensées déconnectées de perceptions.

3. Pour une présentation de ces concepts, voir Rabatel 2000a.

4. Dans les exemples suivants, sauf mention contraire, le PDV est en italiques, le focalisateur en majuscules et le procès de perception et/ou le procès mental est en caractères gras.

5. Il n'y a pas d'obstacle majeur à ce que nous analysions des exemples empruntés à des œuvres étrangères traduites en français. Ce qui nous intéresse, ce sont les marques du PDV en français, 
sans que nous nous préoccupions du texte source. Cela dit, la question de la traduction est tout entière traversée par notre problématique (cf. Danon-Boileau et Bouscaren et SimoninGrumbach, in Langages $\left.n^{\circ} 73,1984\right)$ : il s'agit de la détermination du choix de formes du premier ou du second plans, en français, alors que la langue source (anglais ou allemand, etc.) utilise un tiroir temporel qui peut s'accommoder de deux visées différentes; et réciproquement, du français vers d'autres langues.

6. Un référent est d'autant plus saillant qu'il est doté d'un NP, qu'il est sujet et agent, et qu'il a fait l'objet de mentions préalables. Cf. Schnedecker $1992: 201$ à 210.

7. Aussi parlons nous de PDV du narrateur, perspective pleine qui n'a rien à voir avec une absence de focalisation ou une somme de focalisations variables : cf. Rabatel 1997, chap. 3 et 12 , et Rabatel 1998.

8. Cf. Rabatel $1998: 172$.

9. S. Marnette écrit exactement le contraire : le « rapport narratif d'une action » (cf. "Jeanne regarda par la fenêtre ») indique un "contrôle en apparence total du narrateur »; ce RNA s'oppose au DDL («absence de contrôle apparent du narrateur»), avec, comme étapes intermédiaires, le psycho-récit, le DI, DIL et DD (1998 : 118s). Ce classement repose sur le critère de la présence d'un ou deux locuteurs. Si l'on intègre la problématique de l'énonciateur, ce classement est discutable, car il peut y avoir un autre mode d'autonomisation du personnage, qui ne passe pas nécessairement par l'activité du locuteur... Si le RNA est seulement prédiqué, on peut donner raison à Marnette ; si la perception est représentée (ce cas n'est pas répertorié par S. M.), alors il en va différemment. Fondamentalement, ces différences d'approches invitent à reprendre la question du discours rapporté, à la lumière du PDV : ce sera l'objet de publications ultérieures.

10. Cf. Rabatel « Quand voir c'est (faire) penser ». Actes du colloque international de Lyon sur les relations intersémiotiques. Décembre 1999 (à paraître).

11. Cf. Rabatel 1998 : chap. 4.

12. Cf. Les Mémoires d'outre-tombe, les Mémoires de guerre de De Gaulle, La paille et le grain, de F. Mitterrand, ou les récents ouvrages de Gorbatchev.

\section{RÉSUMÉS}

Cet article analyse des exemples attestés de narrations dans lesquels des indices linguistiques divergents coréfèrent à des sources énonciatives distinctes, et produisent des conflits de perspective narrative entre le point de vue du narrateur et le point de vue du personnage. Loin de devoir être neutralisées, ces perspectives gagnent à être articulées : c'est pourquoi l'on plaide contre les lectures réductrices et l'applicationnisme, pour le plus grand profit de l'interprétation des textes.

This article analyses some examples in wich linguistic markers are referring to different utterers, either the narrator or the actor. The reader is also in front of conflicts between narratorial and actorial points of view. Instead of concluding the neutralization of narrative perspectives, we will try to analyse those conflicts as signals for an interpretation which refuses reductive lectures of narrative discourses. 
AUTEUR

ALAIN RABATEL

IUFM de Lyon

UMR CNRS 5612, GRIC-2 Université de Lyon II

Chercheur associé INRP 